\title{
ELLIPTIC EQUATIONS WITH COMPACTLY SUPPORTED SOLUTIONS
}

\author{
Orazio Arena and Cristina Giannotti
}

\begin{abstract}
For any $p \in(1,2)$ and arbitrary $f \in L^{p}\left(\mathbb{R}^{2}\right)$ with compact support, it is proved that there exists a pair $(L, u)$, with $L$ second order uniformly elliptic operator and $u \in W_{0}^{2, p}\left(\mathbb{R}^{2}\right)$ such that $L u=f$ a.e. in $\mathbb{R}^{2}$
\end{abstract}

\section{Introduction}

Let $L$ be a second order uniformly elliptic operator with bounded measurable coefficients in $\mathbb{R}^{2}$ of the form

$$
L:=a^{11} \partial_{x x}+2 a^{12} \partial_{x y}+a^{22} \partial_{y y}
$$

When $u \in W^{2,2}\left(\mathbb{R}^{2}\right)$ is a solution of the equation $L u=f$ for a compactly supported function $f$, in general, one cannot expect that $u$ also has compact support.

On the other hand, for the case when $p$ is small enough so that the a priori bounds of K. Astala, T. Iwaniec, G. Martin [1] do not hold, Buonocore and Manselli proved in [3] that there exists an operator $L$ (of the above form and with first order terms) and a non trivial $u \in W^{2, p}\left(\mathbb{R}^{2}\right)$ with compact support satisfying the equation $L u=0$ a.e. (see [3]). A similar example in $\mathbb{R}^{3}$ has been constructed in [4].

In this paper, we consider the corresponding question on compactly supported solutions for the non-homogeneous equation $L u=f$ and prove that:

For any given $p \in(1,2)$ and $f \in L^{p}\left(\mathbb{R}^{2}\right)$ with compact support, there exist an operator $L$ of the form (1.1) and a function $u \in W_{0}^{2, p}\left(\mathbb{R}^{2}\right)$ satisfying $L u=f$ a.e. in $\mathbb{R}^{2}$.

The proof basically follows arguments in [6]. In that paper, the authors considered the homogeneous equation $L u=0$ and proved that, given two arbitrary functions $f^{(0)}$ and $f^{(1)}$ on the boundary $\partial D$ of the unit disk $D \subset \mathbb{R}^{2}$, there exists a function $u$ and a second order uniformly elliptic operator $L$ of the form (1.1) so that $L u=0$ in $D,\left.u\right|_{\partial D}=f^{(0)}$ and $\left.\frac{\partial u}{\partial n}\right|_{\partial D}=f^{(1)}$.

Received May 27, 2012; Revised September 30, 2012.

2010 Mathematics Subject Classification. Primary 35B60; Secondary 35J15.

Key words and phrases. second order elliptic equations, compactly supported solutions. 
Here, the construction starts by taking a function $u$ satisfying $\Delta u=f$ a.e. in the unit disk $D$ of the form $u=w+\tilde{u}$, where $w$ is the solution to the Dirichlet problem $\Delta w=f$ in $D, w=0$ on $\partial D$ and $\tilde{u}$ is the sum of a series of Green functions with poles in a countable subset $\mathcal{N}$ of $D$ with no accumulation points in $D$. Such a function $u$ assumes the boundary conditions $\left.u\right|_{\partial D}=0$, $\left.\frac{\partial u}{\partial n}\right|_{\partial D}=0$ in a suitably generalized sense and its existence follows from a result in [5]. After this, following the method used in [6], we modify the function $u$ and the operator $\Delta$ in suitably chosen disks centered at the points of $\mathcal{N}$ and we obtain an elliptic operator $L$ of the form (1.1) and a function $u^{\prime} \in W^{2, p}(D)$ satisfying all required properties.

The paper is organized as follows: In $\S 2$, we recall notations and results of [6] and determine the previously described function $u=w+\tilde{u}$ (Lemma 2.2). In $\S 3$ we outline the modifying procedure for $u$ and $\Delta$ and in $\S 4$, we prove the main result.

\section{Notations and preliminary results}

In what follows, we identify $\mathbb{R}^{2}$ with $\mathbb{C}$ and, for any $r>0$, we denote by $D(\mathfrak{a}, r)$ the open disk centered at $\mathfrak{a} \in \mathbb{C}$ with radius $r$. The unit disk $D(0,1)$ will be simply denoted by $D$.

$\mathcal{L}_{\alpha}$ is the family of linear second order uniformly elliptic operators with bounded measurable coefficients in $D$ of the form (1.1) with lower ellipticity constant $\alpha>0$ and upper ellipticity constant $1 / \alpha$.

Given $W^{2, p}(D)$, the Sobolev space of functions in $L^{p}(D)$ with second derivatives in $L^{p}(D), p>1$, for any $v \in W^{2, p}(D)$, we denote by $\left.v\right|_{\partial D}$ and $\left.\frac{\partial v}{\partial n}\right|_{\partial D}$ the traces on $\partial D$ of $v$ and of $\frac{\partial v}{\partial r}$, respectively. We also denote by $W_{0}^{2, p}(D)$ the closure of $C_{0}^{\infty}(D)$ in $W^{2, p}(D)$, i.e., the class of $v \in W^{2, p}(D)$ such that $\left.v\right|_{\partial D}=0$ and $\left.\frac{\partial v}{\partial n}\right|_{\partial D}=0$.

Let us recall some notations and definitions from [6].

For any real number $\gamma \geq 0$, we denote by $\mathbb{A}^{(\gamma)}$ the Banach space of realvalued functions, defined on $\partial D$, of the form

$$
f\left(e^{i \theta}\right)=\frac{a_{0}}{2}+\sum_{n=1}^{\infty}\left(a_{n} \cos n \theta+b_{n} \sin n \theta\right)
$$

with Fourier coefficients $a_{n}, b_{n}$ such that

$$
\left\{n^{\gamma} a_{n}\right\},\left\{n^{\gamma} b_{n}\right\} \in \ell^{1}
$$

and norm defined by $\|f\|_{\mathbb{A}^{(\gamma)}}=\left\|\left\{n^{\gamma} a_{n}\right\}\right\|_{\ell^{1}}+\left\|\left\{n^{\gamma} b_{n}\right\}\right\|_{\ell^{1}}$.

Also, for any $\gamma \geq 0$, let $\sigma$ be a fixed constant, $0<\sigma<\frac{1}{2}$, depending on $\gamma$ such that

$$
\sum_{p=1}^{\infty}(2 p+1)^{\gamma} \sigma^{2 p}<1
$$


and let $\zeta_{2 n, l}^{(j)}(n \in \mathbb{N}, l=0, \ldots, 2 n-1, j=1,2)$ be the $4 n$-th roots of $\sigma^{4}$ ordered as follows:

$$
\begin{gathered}
\zeta_{2 n, l}^{(1)}=\sigma^{\frac{1}{n}} e^{-\frac{\pi}{n} l i}, \quad l=0, \ldots, 2 n-1, \\
\zeta_{2 n, l}^{(2)}=\sigma^{\frac{1}{n}} e^{-\left(-\frac{\pi}{2 n}+\frac{\pi}{n} l\right) i}, \quad l=0, \ldots, 2 n-1 .
\end{gathered}
$$

Let us denote by $\mathcal{N}:=\left\{\mathfrak{a}_{\nu}\right\}_{\nu \geq 0}$ the sequence given by 0 and the points $\zeta_{2 n, l}^{(j)}$ ordered in the following way:

$$
\mathfrak{a}_{0}=0, \quad \mathfrak{a}_{\nu}=\zeta_{2 n, l}^{(j)} \quad \text { if } \nu=1+2(n-1) n+2(j-1) n+l
$$

for any $n \in \mathbb{N}, l=0, \ldots, 2 n-1, j=1,2$. Notice that $\mathcal{N}$ has no limit points in $D$.

We also set

$$
m_{\nu}:=\frac{1}{2} \min _{\mu \neq \nu}\left|\mathfrak{a}_{\mu}-\mathfrak{a}_{\nu}\right|
$$

(indeed, one may alternatively consider constants $m_{\nu}:=\epsilon \min _{\mu \neq \nu}\left|\mathfrak{a}_{\mu}-\mathfrak{a}_{\nu}\right|$ for any other fixed $\left.\epsilon \in\left(0, \frac{1}{2}\right]\right)$. Notice that as $1+2 n(n-1) \leq \nu \leq 2 n^{2}+2 n$, for any fixed value of $\nu$, the corresponding value of $n$ satisfies the inequalities

$$
\frac{-1+\sqrt{1+2 \nu}}{2} \leq n \leq \frac{1+\sqrt{2 \nu-1}}{2} .
$$

Since $\left|\mathfrak{a}_{\nu}\right|=\sigma^{\frac{1}{n}}$, it follows that $m_{\nu} \geq \frac{\sigma^{\frac{1}{n+1}}-\sigma^{\frac{1}{n}}}{2}=\frac{1}{2} e^{\tilde{x}} \frac{\ln (1 / \sigma)}{n(n+1)}$ for some $\tilde{x} \in$ $\left(\frac{1}{n} \ln \sigma, \frac{1}{n+1} \ln \sigma\right)$ and hence

$$
m_{\nu} \geq \frac{\sigma \ln (1 / \sigma)}{2(n+1)^{2}} \geq \frac{2 \sigma \ln (1 / \sigma)}{(3+\sqrt{2 \nu-1})^{2}} \geq \frac{C(\sigma)}{\nu} .
$$

Notice that the set

$$
D_{o}=D \backslash \cup_{\nu=0}^{\infty} \bar{D}\left(\mathfrak{a}_{\nu}, \frac{2}{3} m_{\nu}\right)
$$

is an open non-empty subset of $D$.

Let $G(z, \zeta)$ be the Green function for the Laplace operator in $D$ with pole $\zeta:$

$$
G(z, \zeta)=-\frac{1}{2 \pi} \ln \left|\frac{z-\zeta}{1-z \bar{\zeta}}\right|, \quad z \neq \zeta .
$$

The following result is proved in [5].

Fact 2.1. Let $\gamma>0$ and $\sigma$ be as above and $1<p<2$. Given $f^{(1)} \in \mathbb{A}^{(\gamma)}$, there exist $a_{0} \in \mathbb{R}$, two sequences $\left\{\alpha_{n}\right\},\left\{\beta_{n}\right\}$ and a constant $K>0$ (depending on $\gamma, p$ only) such that:

(a) $\left|a_{0}\right|+\left\|(\cdot)^{\gamma} \alpha .\right\|_{\ell^{1}}+\left\|(\cdot)^{\gamma} \beta.\right\|\left\|_{\ell^{1}} \leq K\right\| f^{(1)} \|_{\mathbb{A}^{(\gamma)}} ;$ 
(b) The function

$$
\begin{aligned}
\widetilde{u}(z)= & -\pi a_{0} G(z, 0) \\
& -\pi \sum_{n=1}^{\infty} \frac{\alpha_{n}}{2 \sigma n} \sum_{p=0}^{n-1}\left[G\left(z, \zeta_{2 n, 2 p}^{(1)}\right)-G\left(z, \zeta_{2 n, 2 p+1}^{(1)}\right)\right] \\
& -\pi \sum_{n=1}^{\infty} \frac{\beta_{n}}{2 \sigma n} \sum_{p=0}^{n-1}\left[G\left(z, \zeta_{2 n, 2 p}^{(2)}\right)-G\left(z, \zeta_{2 n, 2 p+1}^{(2)}\right)\right]
\end{aligned}
$$

is harmonic in $D \backslash \mathcal{N}$; $\widetilde{u}$ belongs to $L^{p}(D)$ with its first derivatives and

$$
\|\widetilde{u}\|_{L^{p}(D)}+\|D \widetilde{u}\|_{L^{p}(D)} \leq K\left\|f^{(1)}\right\|_{\mathbb{A}^{(\gamma)}} ;
$$

(c) For $N \in \mathbb{N}$, the partial sums of $\widetilde{u}$ defined as

$$
\begin{aligned}
\widetilde{u}^{(N)}(z)= & -\pi a_{0} G(z, 0)-\pi \sum_{n=1}^{N} \frac{\alpha_{n}}{2 \sigma n} \sum_{p=0}^{n-1}\left[G\left(z, \zeta_{2 n, 2 p}^{(1)}\right)-G\left(z, \zeta_{2 n, 2 p+1}^{(1)}\right)\right] \\
& -\pi \sum_{n=1}^{N} \frac{\beta_{n}}{2 \sigma n} \sum_{p=0}^{n-1}\left[G\left(z, \zeta_{2 n, 2 p}^{(2)}\right)-G\left(z, \zeta_{2 n, 2 p+1}^{(2)}\right)\right]
\end{aligned}
$$

have the boundary properties:

i) $\widetilde{u}^{(N)}$ is of class $C^{2}$ in a neighbourhood of $\partial D,\left.\widetilde{u}^{(N)}\right|_{\partial D}=0$, $\left.\frac{\partial \widetilde{u}^{(N)}}{\partial n}\right|_{\partial D} \in \mathbb{A}^{(\gamma)}$

ii) $\widetilde{u}^{(N)}$ converges to $\widetilde{u}$ uniformly on any compact subset of $D \backslash \mathcal{N}$ and

$$
\left\|\left.\frac{\partial \widetilde{u}^{(N)}}{\partial n}\right|_{\partial D}-f^{(1)}\right\|_{\mathbb{A}(\gamma)} \rightarrow 0 .
$$

Now let $f \in L^{p}\left(\mathbb{R}^{2}\right), p>1$, and denote by supp $f$ the support of $f$, i.e., the complement of the greatest open set in which $f=0$ a.e.. For the moment, assume that

$$
\text { supp } f \subset \subset D_{o}=D \backslash \cup_{\nu=0}^{\infty} \bar{D}\left(\mathfrak{a}_{\nu}, \frac{2}{3} m_{\nu}\right)
$$

and let $w \in W^{2, p}(D)$ be the solution to the Dirichlet problem

$$
\left\{\begin{array}{l}
\Delta w=f \text { in } D, \\
w=0 \text { on } \partial D .
\end{array}\right.
$$

Notice that $w$ is harmonic in a neighbourhood of $\partial D$ and hence $\left.\frac{\partial w}{\partial n}\right|_{\partial D}$ belongs to $\mathbb{A}^{(\gamma)}$ for all $\gamma \geq 0$. Thus, the following is an immediate consequence of Fact 2.1, applied with $f^{(1)}=-\left.\frac{\partial w}{\partial n}\right|_{\partial D}$.

Lemma 2.2. Let $\gamma, \sigma, p, f, w$ be as before and $\widetilde{u}$ the function associated to $f^{(1)}:=-\left.\frac{\partial w}{\partial n}\right|_{\partial D}$ as in Fact 2.1. Then 
(a) the function

$$
u=w+\widetilde{u}
$$

is in $W_{\text {loc }}^{2, p}(D \backslash \mathcal{N}) \cap W^{1, p}(D)$ and $\Delta u=f$ a.e. in $D$;

(b) The partial sums $u^{(N)}(z)=w(z)+\widetilde{u}^{(N)}(z)$ converge to u uniformly on compact subsets of $D \backslash \mathcal{N}$, are harmonic in a neighbourhood of $\partial D$ with $\left.u^{(N)}\right|_{\partial D}=0$, and

$$
\left\|\left.\frac{\partial u^{(N)}}{\partial n}\right|_{\partial D}\right\|_{\mathbb{A}(\gamma)} \rightarrow 0 .
$$

Let us write $u$ in the form

$$
u(z)=w(z)+\pi \sum_{\nu=0}^{\infty} \omega_{\nu} G\left(z, \mathfrak{a}_{\nu}\right)
$$

where the coefficients $\omega_{\nu}$ are: $\omega_{0}=-a_{0}$,

$$
\omega_{\nu}=\left\{\begin{array}{l}
(-1)^{l+1} \frac{\alpha_{n}}{2 n \sigma} \quad \text { if } \nu=1+2(n-1) n+l, \\
(-1)^{l+1} \frac{\beta_{n}}{2 n \sigma} \quad \text { if } \nu=1+2(n-1) n+2 n+l
\end{array}\right.
$$

and satisfy $\left\{\nu^{\frac{\gamma}{2}} \omega_{\nu}\right\} \in \ell^{1}$.

Moreover, one can write $u$ as the sum $u=u_{1}+u_{2}$, where

$$
u_{1}(z)=-\frac{1}{2} \sum_{\nu=0}^{\infty} \omega_{\nu} \ln \left|z-\mathfrak{a}_{\nu}\right|
$$

and

$$
u_{2}(z)=w+\frac{1}{2} \sum_{\nu=1}^{\infty} \omega_{\nu}\left(\ln \left|\mathfrak{a}_{\nu}\right|+\ln \left|z-\frac{1}{\overline{\mathfrak{a}}_{\nu}}\right|\right) .
$$

Given $\nu_{0} \in \mathbb{N} \cup\{0\}$, let us define

$$
l^{\left(\nu_{0}\right)}(z):=-\frac{1}{2} \omega_{\nu_{0}} \ln \left|z-\mathfrak{a}_{\nu_{0}}\right|
$$

and

$$
u_{1}^{\left(\nu_{0}\right)}(z):=u_{1}(z)-l^{\left(\nu_{0}\right)}(z)=-\frac{1}{2} \sum_{\nu \neq \nu_{0}} \omega_{\nu} \ln \left|z-\mathfrak{a}_{\nu}\right| .
$$

The following lemma states some properties of these functions.

Lemma 2.3. Let $f \in L^{p}(D)$ with supp $f \subset \subset D_{o}$.

a) $u_{1}$ is harmonic in $D \backslash \mathcal{N}$ and $u_{1}^{\left(\nu_{0}\right)}$ in $(D \backslash \mathcal{N}) \cup\left\{\mathfrak{a}_{\nu_{0}}\right\} ; u_{2}$ is harmonic in $D \backslash$ supp $f$ and $\Delta u_{2}=f$ a.e. in $D$.

b) If $\gamma>2-\frac{2}{p}$, then $u_{2} \in W^{2, p}(D)$. 
c) Let $\nu_{0} \in \mathbb{N} \cup\{0\}$ and $m_{\nu_{0}}$ be as in (2.6). Then there exists a positive constant $A$ such that

$$
\max _{D\left(\mathfrak{a}_{\nu_{0}}, m_{\nu_{0}} / 2\right)}\left|D^{2} u_{2}\right| \leq \frac{A}{m_{\nu_{0}}^{2}}\left(\sum_{\nu=1}^{\infty}\left|\omega_{\nu}\right|+\|f\|_{L^{p}(D)}\right) .
$$

Proof. It is sufficient to prove only (b) and (c). Clearly,

$$
\|w\|_{W^{2, p}(D)} \leq C\|f\|_{L^{p}(D)}
$$

for some constant $C$. Now take a point $z \in \bar{D}$ and observe that

$$
\left|z-\frac{1}{\overline{\mathfrak{a}}_{\nu}}\right| \geq \frac{1}{\left|\mathfrak{a}_{\nu}\right|}-1=\frac{1-\left|\mathfrak{a}_{\nu}\right|}{\left|\mathfrak{a}_{\nu}\right|} .
$$

Since $1-\left|\mathfrak{a}_{\nu}\right|=1-\sigma^{\frac{1}{n}}$ for some $n \in \mathbb{N}$, we have that $1-\left|\mathfrak{a}_{\nu}\right|=e^{\tilde{x}} \frac{1}{n} \ln \frac{1}{\sigma}$ for some $\tilde{x}$ in the interval $\left(\frac{1}{n} \ln \sigma, 0\right)$ and hence, by $(2.7)$,

$$
1-\left|\mathfrak{a}_{\nu}\right| \geq \frac{\sigma \ln 1 / \sigma}{n} \geq \frac{\widetilde{K}}{\sqrt{\nu}}
$$

where $\widetilde{K}$ is a constant depending on $\gamma$. Then from $(2.17)$, one has

$$
\max _{D}\left|u_{2}-w\right| \leq C \sum_{\nu=1}^{\infty}\left|\omega_{\nu}\right|\left(\frac{1}{2}|\log \sigma|+|\log \widetilde{K}|+\frac{1}{2} \log \nu\right)<+\infty .
$$

Moreover, it is not difficult to check that

$$
\begin{aligned}
\left\|D^{2}\left(u_{2}-w\right)\right\|_{L^{p}(D)} & \leq C \sum_{\nu=1}^{\infty}\left|\omega_{\nu}\right|\left\|\left|\cdot-\frac{1}{\overline{\mathfrak{a}}_{\nu}}\right|^{-2}\right\|_{L^{p}(D)} \\
& \leq C \sum_{\nu=1}^{\infty}\left|\omega_{\nu}\right|+C \sum_{\nu=N+1}^{\infty} \nu^{\gamma / 2}\left|\omega_{\nu}\right|\left\|\left|\cdot-\frac{1}{\overline{\mathfrak{a}}_{\nu}}\right|^{\gamma-2}\right\|_{L^{p}\left(D\left(\frac{1}{\overline{\mathfrak{a}}_{\nu}}, 3\right)\right)},
\end{aligned}
$$

where $N$ is chosen sufficiently large such that $\frac{1}{\left|\overline{\mathfrak{a}}_{\nu}\right|} \leq 2$ for $\nu>N$.

Since $2+p(\gamma-2)>0$ and

$$
\left\|\left|\cdot-\frac{1}{\overline{\mathfrak{a}}_{\nu}}\right|^{\gamma-2}\right\|_{L^{p}\left(D\left(\frac{1}{\overline{\mathfrak{a}}_{\nu}}, 3\right)\right)} \leq(2 \pi)^{1 / p}\left(\frac{3^{2+p(\gamma-2))}}{2+p(\gamma-2))}\right)^{1 / p}<+\infty,
$$

(b) follows.

Let us prove (c). As supp $f \subset \subset D_{o}$ and

$$
\left|D_{z}^{2} G(z, \zeta)\right| \leq \frac{C}{|z-\zeta|^{2}}
$$

for some constant $C$, we get

$$
\max _{D\left(\mathfrak{a}_{\nu_{0}}, m_{\nu_{0}} / 2\right)}\left|D^{2} w\right| \leq \max _{D\left(\mathfrak{a}_{\nu_{0}}, m_{\nu_{0}} / 2\right)}\left|\int_{D_{o}} D_{z}^{2} G(z, \zeta) f(\zeta) d \zeta\right| \leq \frac{C}{m_{\nu_{0}}^{2}}\|f\|_{L^{p}(D)} .
$$


Moreover,

$$
\max _{D\left(\mathfrak{a}_{\nu_{0}}, m_{\nu_{0}}\right)}\left|D^{2}\left(u_{2}-w\right)\right| \leq \frac{C}{m_{\nu_{0}}^{2}} \sum_{\nu=1}^{\infty}\left|\omega_{\nu}\right|
$$

and the bound (2.20) follows.

\section{Modifying $u$ and $\Delta$ in neighbourhoods of $\mathfrak{a}_{\nu} \in \mathcal{N}$}

From the results of the previous section, it turns out that we need to modify the function $u$ and the operator $\Delta$ in neighbourhoods of the points $\mathfrak{a}_{\nu} \in \mathcal{N}$ in order to obtain a new function $v$ and a new second order uniformly elliptic operator $L$ of the form (1.1) with the following properties:

i) $v \in C^{1,1}(D \backslash \mathcal{N})$;

ii) $v \in W_{0}^{2, p}(D)$;

iii) $L v=f$ a.e. in $D$.

For a fixed $\nu_{0} \in \mathbb{N} \cup\{0\}$, let

$$
r_{\nu_{0}}=\lambda_{\nu_{0}} m_{\nu_{0}}
$$

with $m_{\nu_{0}}$ as in (2.6) and $\lambda_{\nu_{0}}$ a constant in $(0,1 / 3)$ to be fixed later. Of course, $\bar{D}\left(\mathfrak{a}_{\nu_{0}}, r_{\nu_{0}}\right) \subset D$ and $\bar{D}\left(\mathfrak{a}_{\nu_{0}}, 2 r_{\nu_{0}}\right) \cap \bar{D}\left(\mathfrak{a}_{\nu}, 2 r_{\nu}\right)=\emptyset$ if $\nu \neq \nu_{0}$.

To modify the function $u$ inside the disk $D\left(\mathfrak{a}_{\nu_{0}}, r_{\nu_{0}}\right)$, let us replace the term $l^{\left(\nu_{0}\right)}$ with a smoother function as suggested by the following lemma from [6].

Lemma 3.1. Let $\nu_{0} \in \mathbb{N} \cup\{0\}, 1<p<2,0<2-\frac{2}{p}<h<1$ and consider the function in $D\left(\mathfrak{a}_{\nu_{0}}, r_{\nu_{0}}\right)$ defined by

$$
s^{\left(\nu_{0}\right)}(r)=H_{0}^{\left(\nu_{0}\right)}+H_{1}^{\left(\nu_{0}\right)} r^{h}, \quad r=\left|z-\mathfrak{a}_{\nu_{0}}\right|,
$$

where

$$
H_{0}^{\left(\nu_{0}\right)}=-\frac{\omega_{\nu_{0}}}{2} \ln r_{\nu_{0}}+\frac{\omega_{\nu_{0}}}{2 h}, \quad H_{1}^{\left(\nu_{0}\right)}=-\frac{\omega_{\nu_{0}}}{2 h} r_{\nu_{0}}^{-h}
$$

Then

i) $s^{\left(\nu_{0}\right)}\left(r_{\nu_{0}}\right)=l^{\left(\nu_{0}\right)}\left(r_{\nu_{0}}\right)$ and $\frac{\partial s^{\left(\nu_{0}\right)}}{\partial r}\left(r_{\nu_{0}}\right)=\frac{\partial l^{\left(\nu_{0}\right)}}{\partial r}\left(r_{\nu_{0}}\right)$;

ii) $s^{\left(\nu_{0}\right)}\left(\left|\cdot-\mathfrak{a}_{\nu_{0}}\right|\right)$ belongs to $W^{2, p}\left(D\left(\mathfrak{a}_{\nu_{0}}, r_{\nu_{0}}\right)\right)$ and

$$
\left\|s^{\left(\nu_{0}\right)}\right\|_{L^{p}\left(D\left(\mathfrak{a}_{\nu_{0}}, r_{\nu_{0}}\right)\right)} \leq C\left|\omega_{\nu_{0}}\right| r_{\nu_{0}}^{2 p}\left(1+\left|\ln r_{\nu_{0}}\right|\right)
$$

$$
\left\|\Delta s^{\left(\nu_{0}\right)}\right\|_{L^{p}\left(D\left(\mathfrak{a}_{\nu_{0}}, r_{\nu_{0}}\right)\right)}=C^{\prime}\left|\omega_{\nu_{0}}\right| r_{\nu_{0}}^{\frac{2}{p}-2}
$$

where $C$ and $C^{\prime}$ are constants, both depending only on $p$ and on $h$.

For reader's convenience, we recall its short proof.

Proof. It is enough to prove the last two formulas. By means of (3.1) and (3.2), we have

$$
\left\|s^{\left(\nu_{0}\right)}\right\|_{L^{p}\left(D\left(\mathfrak{a}_{\nu_{0}}, r_{\nu_{0}}\right)\right)} \leq\left|H_{0}^{\left(\nu_{0}\right)}\right| \pi^{1 / p} r_{\nu_{0}}^{2 / p}+\left|H_{1}^{\left(\nu_{0}\right)}\right|(2 \pi)^{1 / p}\left(\int_{0}^{r_{\nu_{0}}} r^{p h+1} d r\right)^{\frac{1}{p}}
$$




$$
\begin{aligned}
& =\pi^{1 / p} \frac{\left|\omega_{\nu_{0}}\right|}{2} r_{\nu_{0}}^{2 / p}\left(\left|\ln r_{\nu_{0}}\right|+\frac{1}{h}+\frac{2^{1 / p}}{h(p h+2)^{1 / p}}\right) \\
& \leq C\left|\omega_{\nu_{0}}\right| r_{\nu_{0}}^{2 / p}\left(1+\left|\ln r_{\nu_{0}}\right|\right) .
\end{aligned}
$$

Moreover, $\Delta s^{\left(\nu_{0}\right)}=\left(s^{\left(\nu_{0}\right)}\right)_{r r}+\frac{\left(s^{\left(\nu_{0}\right)}\right)_{r}}{r}=-\frac{h}{2} \omega_{\nu_{0}} r_{\nu_{0}}^{-h} r^{h-2}$ so that

$$
\begin{aligned}
\left\|\Delta s^{\left(\nu_{0}\right)}\right\|_{L^{p}\left(D\left(\mathfrak{a}_{\nu_{0}}, r_{\nu_{0}}\right)\right)} & =(2 \pi)^{1 / p}\left|\omega_{\nu_{0}}\right| r_{\nu_{0}}^{-h} \frac{h}{2}\left(\int_{0}^{r_{\nu_{0}}} r^{p(h-2)+1} d r\right)^{\frac{1}{p}} \\
& =\frac{(2 \pi)^{1 / p}\left|\omega_{\nu_{0}}\right|(h / 2)}{(p(h-2)+2)^{1 / p}} r_{\nu_{0}}^{\frac{2}{p}-2}
\end{aligned}
$$

Then we have:

Lemma 3.2. Let $p, h, \nu_{0}$ be as in the previous lemma. Set $\Omega=\sum_{\nu=0}^{\infty}\left|\omega_{\nu}\right|+$ $\|f\|_{L^{p}(D)}$ and

$$
\lambda_{\nu_{0}}=\min \left\{\frac{1}{4}, \sqrt{\frac{(1-h)\left|\omega_{\nu_{0}}\right|}{4(4 A+1) \Omega}}\right\}
$$

where $A$ is the constant in the estimate (2.20).

Consider the following function $v^{\left(\nu_{0}\right)}$ on $D\left(\mathfrak{a}_{\nu_{0}}, 2 r_{\nu_{0}}\right)$ :

$$
v^{\left(\nu_{0}\right)}=\left\{\begin{array}{lll}
s^{\left(\nu_{0}\right)}\left(\left|\cdot-\mathfrak{a}_{\nu_{0}}\right|\right)+u_{1}^{\left(\nu_{0}\right)}+u_{2} & \text { in } & D\left(\mathfrak{a}_{\nu_{0}}, r_{\nu_{0}}\right), \\
u & \text { in } & D\left(\mathfrak{a}_{\nu_{0}}, 2 r_{\nu_{0}}\right) \backslash D\left(\mathfrak{a}_{\nu_{0}}, r_{\nu_{0}}\right) .
\end{array}\right.
$$

It turns out that:

(a) $v^{\left(\nu_{0}\right)} \in C^{1,1}\left(D\left(\mathfrak{a}_{\nu_{0}}, 2 r_{\nu_{0}}\right) \backslash\left\{\mathfrak{a}_{\nu_{0}}\right\}\right)$;

(b) $v^{\left(\nu_{0}\right)}$ is harmonic in $D\left(\mathfrak{a}_{\nu_{0}}, 2 r_{\nu_{0}}\right) \backslash D\left(\mathfrak{a}_{\nu_{0}}, r_{\nu_{0}}\right)$;

(c) $v^{\left(\nu_{0}\right)} \in W^{2, p}\left(D\left(\mathfrak{a}_{\nu_{0}}, 2 r_{\nu_{0}}\right)\right)$.

Moreover, $v^{\left(\nu_{0}\right)}$ satisfies a second order, uniformly elliptic equation $L v^{\left(\nu_{0}\right)}=0$ with bounded measurable coefficients in $D\left(\mathfrak{a}_{\nu_{0}}, 2 r_{\nu_{0}}\right)$ and lower ellipticity constant $\frac{1}{2}(1-h)$.

Proof. Statement (a) follows by Lemma 3.1(i) and from the fact that $v^{\left(\nu_{0}\right)}$ has second order derivatives bounded in every compact subset of $D\left(\mathfrak{a}_{\nu_{0}}, 2 r_{\nu_{0}}\right) \backslash$ $\left\{\mathfrak{a}_{\nu_{0}}\right\}$. Statement (b) is clear and in regard to (c), it is enough to use Lemma 3.1(ii).

As far as the last claim is concerned, by known facts (see e.g. [2], Ch. 6), one needs to verify the existence of a number $q \in(0,1)$ such that

$$
\left|\frac{\left(v^{\left(\nu_{0}\right)}\right)_{z \bar{z}}}{\left(v^{\left(\nu_{0}\right)}\right)_{z z}}\right| \leq q \quad \text { in } D\left(\mathfrak{a}_{\nu_{0}}, r_{\nu_{0}}\right)
$$


Let us prove that (3.6) holds true with $q=h$. Indeed, recalling that $u_{1}^{\left(\nu_{0}\right)}$ and $u_{2}$ are harmonic in $D\left(\mathfrak{a}_{\nu_{0}}, r_{\nu_{0}}\right)$, one may write

$$
\left|\frac{\left(v^{\left(\nu_{0}\right)}\right)_{z \bar{z}}}{\left(v^{\left(\nu_{0}\right)}\right)_{z z}}\right|=\left|\frac{\left(s^{\left(\nu_{0}\right)}\left(\left|\cdot-\mathfrak{a}_{\nu_{0}}\right|\right)\right)_{z \bar{z}}}{\left(s^{\left(\nu_{0}\right)}\left(\left|\cdot-\mathfrak{a}_{\nu_{0}}\right|\right)+u_{1}^{\left(\nu_{0}\right)}+u_{2}\right)_{z z}}\right| .
$$

On the other hand, by (3.1), (3.2) and using polar coordinates with origin $\mathfrak{a}_{\nu_{0}}$,

$$
\begin{aligned}
\left(s^{\left(\nu_{0}\right)}\left(\left|\cdot-\mathfrak{a}_{\nu_{0}}\right|\right)\right)_{z z} & =\frac{\omega_{\nu_{0}}}{4}\left(1-\frac{h}{2}\right)\left(\frac{r}{r_{\nu_{0}}}\right)^{h}\left(z-\mathfrak{a}_{\nu_{0}}\right)^{-2}, \\
\left(s_{\nu_{0}}\left(\left|\cdot-\mathfrak{a}_{\nu_{0}}\right|\right)\right)_{z \bar{z}} & =-\frac{\omega_{\nu_{0}}}{8} h\left(\frac{r}{r_{\nu_{0}}}\right)^{h} r^{-2} .
\end{aligned}
$$

Then by easy calculations,

$$
\begin{aligned}
\left|\frac{\left(v^{\left(\nu_{0}\right)}\right)_{z \bar{z}}}{\left(v^{\left(\nu_{0}\right)}\right)_{z z}}\right| & =\left|\frac{\omega_{\nu_{0}} h r^{-2}\left(z-\mathfrak{a}_{\nu_{0}}\right)^{2}}{2 \omega_{\nu_{0}}\left(1-\frac{h}{2}\right)+8\left(u_{1}^{\left(\nu_{0}\right)}+u_{2}\right)_{z z}\left(\frac{r}{r_{\nu_{0}}}\right)^{-h}\left(z-\mathfrak{a}_{\nu_{0}}\right)^{2}}\right| \\
& =\frac{\left|\omega_{\nu_{0}}\right| h}{\left|\omega_{\nu_{0}}(2-h)+8\left(u_{1}^{\left(\nu_{0}\right)}+u_{2}\right)_{z z}\left(\frac{r}{r_{\nu_{0}}}\right)^{-h}\left(z-\mathfrak{a}_{\nu_{0}}\right)^{2}\right|} .
\end{aligned}
$$

Now, it is clear that

$$
\left|8\left(u_{1}^{\left(\nu_{0}\right)}+u_{2}\right)_{z z}\left(\frac{r}{r_{\nu_{0}}}\right)^{-h}\left(z-\mathfrak{a}_{\nu_{0}}\right)^{2}\right| \leq 8 r_{\nu_{0}}^{2} \max _{\bar{D}\left(\mathfrak{a}_{\nu_{0}}, r_{\nu_{0}}\right)}\left|\left(u_{1}^{\left(\nu_{0}\right)}+u_{2}\right)_{z z}\right| .
$$

Moreover, since for any $z \in \bar{D}\left(\mathfrak{a}_{\nu_{0}}, r_{\nu_{0}}\right)$ and $\nu \neq \nu_{0}$,

$$
\left|z-\mathfrak{a}_{\nu}\right| \geq\left|\mathfrak{a}_{\nu_{0}}-\mathfrak{a}_{\nu}\right|-\left|z-\mathfrak{a}_{\nu_{0}}\right| \geq m_{\nu_{0}}-r_{\nu_{0}}=\left(1-\lambda_{\nu_{0}}\right) m_{\nu_{0}},
$$

we have

$$
\left|\frac{\partial^{2} u_{1}^{\left(\nu_{0}\right)}}{\partial z^{2}}\right| \leq \frac{1}{4} \sum_{\nu \neq \nu_{0}} \frac{\left|\omega_{\nu}\right|}{\left|z-\mathfrak{a}_{\nu}\right|^{2}} \leq \frac{1}{4} \frac{\Omega}{\left(1-\lambda_{\nu_{0}}\right)^{2} m_{\nu_{0}}^{2}}
$$

and, recalling the bound (2.20) and using (3.5), we get

$$
8 r_{\nu_{0}}^{2} \max _{\bar{D}\left(\mathfrak{a}_{\nu_{0}}, r_{\nu_{0}}\right)}\left|\left(u_{1}^{\left(\nu_{0}\right)}+u_{2}\right)_{z z}\right| \leq 8 r_{\nu_{0}}^{2}\left\{\frac{A \Omega}{m_{\nu_{0}}^{2}}+\frac{\Omega}{4\left(1-\lambda_{\nu_{0}}\right)^{2} m_{\nu_{0}}^{2}}\right\} \leq(1-h)\left|\omega_{\nu_{0}}\right| .
$$

Therefore,

$$
\left|\frac{\left(v^{\left(\nu_{0}\right)}\right)_{z \bar{z}}}{\left(v^{\left(\nu_{0}\right)}\right)_{z z}}\right| \leq \frac{\left|\omega_{\nu_{0}}\right| h}{\left|\omega_{\nu_{0}}\right|(2-h)-8\left(r_{\nu_{0}}\right)^{2} \max _{\bar{D}\left(\mathfrak{a}_{\nu_{0}}, r_{\nu_{0}}\right)}\left|\left(u_{1}^{\left(\nu_{0}\right)}+u_{2}\right)_{z z}\right|} \leq h
$$

and (3.6) holds true for $q=h$. 


\section{The main theorem}

Now we are ready to prove our main result under the hypothesis that supp $\subset \subset \subset D_{o}=D \backslash \cup_{\nu=0}^{\infty} \bar{D}\left(\mathfrak{a}_{\nu}, \frac{2}{3} m_{\nu}\right)$.

Theorem 4.1. Let $1<p<2,2-\frac{2}{p}<h<1$ and $f \in L^{p}(D)$ with supp $\subset \subset$ $D_{o}$. Then there exist a function $v \in W_{0}^{2, p}(D)$ and a uniformly elliptic operator $L$ of the form (1.1) with bounded measurable coefficients in $D$ and lower ellipticity constant $\frac{1-h}{2}$ such that $L v=f$ a.e. in $D$.

Proof. In what follows, we will denote by the same letter $C$ different constants. Choose $\gamma>4(p-1)$ and notice that since $\gamma>2-\frac{2}{p}$, Lemma 2.3 holds true. For any $\nu \in \mathbb{N} \cup\{0\}$, denote by $D_{\nu}$ the disk of center $\mathfrak{a}_{\nu}$ and radius $r_{\nu}=\lambda_{\nu} m_{\nu}$, where $\lambda_{\nu}$ is given by (3.5). Let

$$
v:= \begin{cases}u & \text { in } D \backslash \bigcup_{\nu=0}^{\infty} D_{\nu}, \\ v^{(\nu)}=s^{(\nu)}+u_{1}^{(\nu)}+u_{2} & \text { in } D_{\nu} \text { for all } \nu=0,1 \ldots\end{cases}
$$

Then $v$ satisfies $\Delta v=f$ in $D \backslash \bigcup_{\nu=0}^{\infty} D_{\nu}$ and, by Lemma 3.2, it solves an elliptic equation $L v=0$, i.e., $L v=f$, in each $D_{\nu}$ with ellipticity constant $\frac{1}{2}(1-h)$. By the same lemma, it is also in $W_{l o c}^{2, p}(D)$. To prove that $v \in W^{2, p}(D)$, it is sufficient to show that $v \in L^{p}(D)$ and $\Delta v \in L^{p}(D)$. First of all, one has

$$
\|v\|_{L^{p}(D)} \leq\|u\|_{L^{p}(D)}+\frac{1}{2} \sum_{\nu=0}^{\infty}\left|\omega_{\nu}\right|\left\|\log \left(\left|\cdot-\mathfrak{a}_{\nu}\right|\right)\right\|_{L^{p}\left(D_{\nu}\right)}+\sum_{\nu=0}^{\infty}\left\|s_{\nu}\right\|_{L^{p}\left(D_{\nu}\right)} .
$$

Now by Lemma 2.3, $\|u\|_{L^{p}(D)}<+\infty$; moreover,

$$
\sum_{\nu=0}^{\infty}\left|\omega_{\nu}\right||| \log \left(\left|\cdot-\mathfrak{a}_{\nu}\right|\right) \|_{L^{p}\left(D_{\nu}\right)} \leq C \sum_{\nu=0}^{\infty}\left|\omega_{\nu}\right|<+\infty .
$$

In addition, by (3.3) of Lemma 3.1,

$$
\sum_{\nu=0}^{\infty}\left\|s_{\nu}\right\|_{L^{p}\left(D_{\nu}\right)} \leq C \sum_{\nu=0}^{\infty}\left|\omega_{\nu}\right| r_{\nu}^{2 p}\left(1+\left|\log \left(r_{\nu}\right)\right|\right)<+\infty
$$

since $\left\{\omega_{\nu}\right\} \in \ell^{1}$ and $r_{\nu}^{2 p}\left(1+\left|\log \left(r_{\nu}\right)\right|\right)$ tends to zero as $\nu \rightarrow \infty$. Hence $v \in$ $L^{p}(D)$.

On the other hand, since $\Delta v=f$ in $D \backslash \bigcup_{\nu=0}^{\infty} D_{\nu}$ and by (3.4) of Lemma 3.1 ,

$$
\begin{aligned}
\|\Delta v\|_{L^{p}(D)}^{p} & \leq\|f\|_{L^{p}(D)}^{p}+\sum_{\nu=0}^{\infty}\left\|\Delta s_{\nu}\right\|_{L^{p}\left(D_{\nu}\right)}^{p} \\
& \leq\|f\|_{L^{p}(D)}^{p}+C \sum_{\nu=0}^{\infty}\left|\omega_{\nu}\right|^{p} r_{\nu}^{2(1-p)} .
\end{aligned}
$$


Now from (3.5), we may write $\lambda_{\nu}^{2} \leq C\left|\omega_{\nu}\right|$ and using (2.8) for sufficiently large $\nu$, we have

$$
\left|\omega_{\nu}\right|^{p} r_{\nu}^{2(1-p)}=\left|\omega_{\nu}\right|^{p}\left(\lambda_{\nu}^{2} m_{\nu}^{2}\right)^{(1-p)} \leq \mathrm{C} \frac{\left|\omega_{\nu}\right|}{\nu^{2(1-p)}}=\mathrm{C}\left(\left|\omega_{\nu}\right| \nu^{\frac{\gamma}{2}}\right) \nu^{-\frac{\gamma}{2}+2(p-1)} .
$$

Since $\left\{\nu^{\gamma / 2} \omega_{\nu}\right\} \in \ell^{1}$ and $2(p-1)-\gamma / 2 \leq 0$, it follows that the series

$$
\sum_{\nu=0}^{\infty}\left|\omega_{\nu}\right|^{p} r_{\nu}^{2(1-p)}
$$

is convergent and hence that $\|\Delta v\|_{L^{p}(D)}^{p}<+\infty$.

To conclude, we need to prove that $\left.v\right|_{\partial D}=0$ and $\left.\frac{\partial v}{\partial n}\right|_{\partial D}=0$.

For any $N=0,1, \ldots$, we set

$$
v^{(N)}:= \begin{cases}u^{(N)} & \text { in } D \backslash \bigcup_{\nu=0}^{N} D_{\nu}, \\ u^{(N)}+s^{(\nu)}-l^{(\nu)} & \text { in } D_{\nu}, \nu=0,1, \ldots, N,\end{cases}
$$

where $u^{(N)}$ is the partial sum defined in Lemma 2.3. The function $v^{(N)}$ coincides with $u^{(N)}$ in a neighbourhood of $\partial D$ and hence it is regular up to the boundary. In particular,

$$
\left.v^{(N)}\right|_{\partial D}=0 \quad \text { and } \quad \lim _{N \rightarrow \infty}\left\|\left.\frac{\partial v^{(N)}}{\partial n}\right|_{\partial D}\right\|_{\mathbb{A}(\gamma)}=0 .
$$

Moreover, $v^{(N)}$ converges to $v$ in $L^{p}(D)$ : In fact,

$$
\begin{gathered}
\left\|v^{(N)}-v\right\|_{L^{p}(D)}^{p} \leq\left\|v^{(N)}-v\right\|_{L^{p}\left(D \backslash \bigcup_{\nu=N+1}^{\infty} D_{\nu}\right)}^{p}+\sum_{\nu=N+1}^{\infty}\left\|v^{(N)}-v\right\|_{L^{p}\left(D_{\nu}\right)}^{p}, \\
\left\|v^{(N)}-v\right\|_{L^{p}\left(D \backslash \bigcup_{\nu=N+1}^{\infty} D_{\nu}\right)} \leq \pi \sum_{\nu=N+1}^{\infty}\left|\omega_{\nu}\right|\left\|G\left(\cdot, \mathfrak{a}_{\nu}\right)\right\|_{L^{p}(D)} \\
\leq C(p) \sum_{\nu=N+1}^{\infty} \mid \omega_{\nu} \stackrel{N \rightarrow \infty}{\longrightarrow} 0
\end{gathered}
$$

and

$$
\begin{aligned}
\sum_{\nu=N+1}^{\infty}\left\|v^{(N)}-v\right\|_{L^{p}\left(D_{\nu}\right)} \leq & \pi \sum_{\nu=N+1}^{\infty}\left|\omega_{\nu}\right|\left\|G\left(\cdot, \mathfrak{a}_{\nu}\right)\right\|_{L^{p}(D)}+\sum_{\nu=N+1}^{\infty}\left\|s_{\nu}\right\|_{L^{p}\left(D_{\nu}\right)}+ \\
& +\frac{1}{2} \sum_{\nu=N+1}^{\infty}\left|\omega_{\nu}\right|\left\|\ln \left|z-\mathfrak{a}_{\nu}\right|\right\|_{L^{p}(D)} \\
\leq & \mathrm{C} \sum_{\nu=N+1}^{\infty}\left|\omega_{\nu}\right|+\sum_{\nu=N+1}^{\infty}\left\|s^{\nu}\right\|_{L^{p}\left(D_{\nu}\right)} \stackrel{N \rightarrow \infty}{\longrightarrow} 0 .
\end{aligned}
$$


In addition,

$$
\left\|\Delta v^{(N)}-\Delta v\right\|_{L^{p}(D)} \leq \sum_{\nu=N+1}^{\infty}\left\|\Delta s^{\nu}\right\|_{L^{p}\left(D_{\nu}\right)} \stackrel{N \rightarrow \infty}{\longrightarrow} 0
$$

since the series $\sum_{\nu=0}^{\infty}\left\|\Delta s_{\nu}\right\|_{L^{p}\left(D_{\nu}\right)}$ converge.

From this it follows that $v^{(N)}$ converges to $v$ in $W^{2, p}(D)$, and hence that $\left.v^{(N)}\right|_{\partial D}$ tends to $\left.v\right|_{\partial D}$ in $W^{2-1 / p, p}(\partial D)$ and $\left.\frac{\partial v^{(N)}}{\partial n}\right|_{\partial D}$ tends to $\left.\frac{\partial v}{\partial n}\right|_{\partial D}$ in $W^{1-1 / p, p}(\partial D)$. This implies that $\left.v\right|_{\partial D}=0$ and $\left.\frac{\partial v}{\partial n}\right|_{\partial D}=0$.

Finally, let us remove the previous restriction on the support of $f$.

Theorem 4.2. Let $1<p<2,2-\frac{2}{p}<h<1$ and $f \in L^{p}\left(\mathbb{R}^{2}\right)$ with compact support. Then there exist a function $v \in W_{0}^{2, p}\left(\mathbb{R}^{2}\right)$ and a uniformly elliptic operator $L$ with bounded and measurable coefficients and lower ellipticity constant $\frac{1-h}{2}$ such that $L v=f$ a.e. in $\mathbb{R}^{2}$.

Proof. Assume supp $f \subset D(0, R)$ and let $z_{o} \in D_{o}$ and $\rho>0$ be such that $\bar{D}\left(z_{o}, \rho\right) \subset D_{o}$. Then $\widetilde{f}\left(z^{\prime}\right):=\left(\frac{\rho}{R}\right)^{2} f\left(\frac{R}{\rho}\left(z^{\prime}-z_{o}\right)\right)$ satisfies supp $\tilde{f} \subset D\left(z_{o}, \rho\right)$ and by Theorem 4.1, there exist a function $\widetilde{v} \in W_{0}^{2, p}(D)$ and a uniformly elliptic operator $\widetilde{L}$ of the form $\widetilde{L}:=\widetilde{a}^{11}\left(z^{\prime}\right) \partial_{x^{\prime} x^{\prime}}+2 \widetilde{a}^{12}\left(z^{\prime}\right) \partial_{x^{\prime} y^{\prime}}+\widetilde{a}^{22}\left(z^{\prime}\right) \partial_{y^{\prime} y^{\prime}}$ with lower ellipticity constant $\frac{1-h}{2}$ such that $\widetilde{L} \widetilde{v}=\widetilde{f}$ a.e. in $D$.

Now, let $v(z):=\widetilde{v}\left(z^{\prime}\right)=\widetilde{v}\left(z_{o}+\frac{\rho}{R} z\right)$ and $L:=a^{11}(z) \partial_{x x}+2 a^{12}(z) \partial_{x y}+$ $a^{22}(z) \partial_{y y}$ with $a^{i j}(z):=\widetilde{a}^{i j}\left(z^{\prime}\right)=\widetilde{a}^{i j}\left(z_{o}+\frac{\rho}{R} z\right)$ in $D\left(-(R / \rho) z_{o}, R / \rho\right)$ and $L=\Delta$, otherwise. Then $v \in W_{0}^{2, p}\left(\mathbb{R}^{2}\right), L$ is uniformly elliptic with the same ellipticity constant of $\widetilde{L}$ and $L v=f$ a.e. in $\mathbb{R}^{2}$.

Remark 4.3. Let $p, f, v, L$ be as in Theorem 4.2. By classical results on second order elliptic equations and elliptic first order system (see e.g. [2]), one has that the function $w:=v_{x}-i v_{y}$ belongs to $W_{0}^{1, p}(\mathbb{C})$ and satisfies a complex uniformly elliptic first order system of the form

$$
w_{\bar{z}}=\mu w_{z}+\nu \bar{w}_{\bar{z}}+\gamma \quad \text { in } \mathbb{C}
$$

with $\mu=\mu(z), \nu=\nu(z)$ and $\gamma=\gamma(z)$, complex-valued functions, such that $|\mu|+|\nu| \leq k<1$ and $|\gamma| \leq k^{\prime}$.

\section{References}

[1] K. Astala, T. Iwaniec, and G. Martin, Pucci's conjecture and the Alexandrov inequality for elliptic PDEs in the plane, J. Reine Angew. Math. 591 (2006), 49-74.

[2] L. Bers, F. John, and M. Schechter, Partial Differential Equations, Interscience, 1964.

[3] P. Buonocore and P. Manselli, Solutions to two dimensional, uniformly elliptic equations, that lie in Sobolev spaces and have compact support, Rend. Circ. Mat. Palermo (2) 51 (2002), no. 3, 476-484.

[4] C. Giannotti, A compactly supported solution to a three-dimensional uniformly elliptic equation without zero order term, J. Differential Equations 201 (2004), no. 2, 234-249. 
[5] C. Giannotti and P. Manselli, Expansions with Poisson kernels and related topics, Proc. Edinb. Math. Soc. (2) 53 (2010), no. 1, 153-173.

[6] _ On elliptic extensions in the disk, Potential Anal. 33 (2010), no. 3, 249-262.

[7] T. H. Wolff, Some constructions with solutions of variable coefficient elliptic equations, J. Geom. Anal. 3 (1993), no. 5, 423-511.

\section{Orazio Arena}

Dipartimento di Costruzioni e Restauro

Università di Firenze

Piazza Brunelleschi, 6

I-50121 Firenze, ItALy

E-mail address: arena@unifi.it

Cristina Giannotti

Scuola di Scienze e Tecnologie

Università di CAMERINO

Via Madonna delle Carceri

I- 62032 Camerino (Macerata), Italy

E-mail address: cristina.giannotti@unicam.it 